\title{
Lung cancer screening: national consensus is an important step toward implementation
}

The author declares no financial disclosure

Lung cancer is the primary cause of cancer related death in the world, in Europe and also in Poland. In spite of significant improvements in diagnosis and treatment over the last $10-20$ years only modest increases in 5-year survival are being achieved. In Poland 5-year survival has increased from 11.4 to $13.4 \%$, which is comparable to other European countries, but lower than the $18.7 \%$ achieved in the US and $30.1 \%$ in Japan. One of the main reasons for this is that lung cancer still in clinical practice in Europe is diagnosed in symptomatic and therefore mostly in advanced stages of the disease. Early diagnosis of lung cancer by low dose CT (LDCT) screening has the potential to fundamentally change this scenario. The results from the randomized National Lung Screening Trial (NLST) in the United States were remarkably clear to show an effect of CT screening on both lung cancer and all cause mortality. However the observed effect was the result of only three annual screenings. Therefore it is important to realize that the magnitude of the mortality reduction by continued annual CT screening over many years most likely would exceed that observed in the NLST trial. During the last 15 years considerable evidence and experience with this method has been established. Poland has made important contributions to this field and almost 50.000 individuals have so far participated in CT screening studies in Polish centers. It is therefore timely and appropriate that dr. Witold Rzyman and co-authors from 6 major centers in Poland, in this issue of ARM can publish a "Consensus statement on a screening programme for the detection of early lung cancer in Poland".

The statement is very comprehensive and covers all the issues that need to be taken into account before implementation. It provides the relevant background knowledge on diagnosis and treatment of lung cancer, and the risks and expected outcomes of early detection of lung cancer using LDCT. The technical and organizational requirements for implementation of CT screening (equipment, radiation dose, nodule evaluation, quality control etc.) is described and discussed, and also the qualifications and performance of teams conducting the screening, including requirements for radiologists, pathologists and thoracic surgeons involved in the screening. The question of who should be screened is discussed and clear recommendations for inclusion criteria and risk assessment are presented as well as a preliminary estimate of the costs associated with national implementation in Poland.

The paper also emphasizes the need for lung cancer screening to be associated with an active smoking cessation program. A specific pathway for the smoking cessation intervention is described and this is a valuable tool in the implementation process in Poland which will increase the cost effectiveness of the screening program considerably. Lastly the inclusion of additional programmes for the detection of cardiovascular disease (CAC score, Blood Pressure cholesterol) and COPD is described and recommended. Although there are additional costs

Address for correspondence: Jesper Holst Pedersen, Associate Professor, University of Copenhagen, Department of cardiothoracic Surgery, Rigshospitalet, Copenhagen, Denmark, e-mail: Jesper.Holst.Pedersen@regionh.dk DOI: 10.5603/ARM.2018.0001

Received: 22.02.2018

Copyright (C) 2018 PTChP

ISSN 2451-4934 
associated with this, it is expected that these are manageable, and that in all this will increase the cost-effectiveness of the CT screening program further.

The authors are to be congratulated with achieving this national consensus, which is very important for the future discussions on when and how to implement CT screening in Poland. This document will also be important for other countries in Europe as inspiration and guidance for national considerations on how to implement CT screening. 